\title{
EVALUACIÓN DE HABILIDADES PARENTALES, DESDE PROFESIONALES DEL ÁMBITO DEL DERECHO DE FAMILIA
}

OCTAVIO ASTUDILLO MARTíNEZ ; (FUNDACIÓN TIERRA DE ESPERANZA, Centro de Evaluación DAM, Chile) IVANNIA GÁLVEZ GALLARDO ${ }_{2 i}$ (SERVICIO DE PAZ Y JUSTICIA, Programa de Intervención Breve, Chile) CLAUDIO RETAMALES MOLINA A $_{3}$ (FUNDACIÓN TIERRA DE ESPERANZA, Centro de Evaluación DAM, Chile) MAY-LYN ROJAS VALDIVIA ${ }_{4 i}$ (CENTRO DE ATENCIÓN INTEGRAL A VÍCTIMAS DE DELITOS VIOLENTOS, Chile) WILSON SARRIA ÓRDENES 5 (INVESTIGADOR INDEPENDIENTE, Chile)

\begin{abstract}
RESUMEN
La presente investigación tiene como objetivo describir el proceso de evaluación de habilidades parentales, llevado a cabo por los/as profesionales pertenecientes al ámbito del derecho de familia de la ciudad de Antofagasta. Para esto se utilizó una metodología de tipo cualitativa, desde un paradigma interpretativo llevando a cabo entrevistas a expertos en el área, analizando la información desde The Grounded Theory de Glaser \& Strauss. Entre los principales hallazgos, se encuentra que a nivel metodológico destacan como modalidades de evaluación la mirada psicosocial y la psicológico-pericial; y que el Apego y la Empatía surgen como los principales elementos a evaluar en los/as referentes. Así también, en cuanto a las técnicas e instrumentos utilizados destacan la Entrevista Semi-estructurada y el Test de Rorschach. A nivel teórico, los enfoques utilizados son el sistémico, ecológico y clínico-pericial. Por otra parte, los profesionales brindan una mayor valoración a la experiencia por sobre la formación teórica, mencionando como característica de personalidad relevante la empatía, para desarrollar mejor el proceso. Por último, cabe señalar que como principal debilidad del proceso se identifica la falta de estandarización de parámetros, tanto metodológicos como teóricos, para comprender y evaluar las habilidades parentales, tarea que en la actualidad queda a criterio de los/as evaluadores/as, limitando la objetividad; y dificultando la toma de decisión respecto a la habilitación e inhabilitación de un/a referente, afectando el principio de Bienestar Superior del/la Niño/a.
\end{abstract}

PALABRAS CLAVE: Habilidades Parentales, Evaluación, Derecho de Familia.

\section{ABSTRACT}

The aim of this current research is to describe the evaluation process of parental skills, developed by the professionals which are members of the field of Family Right of Antofagasta city. In this research it's been used a qualitative methodology from an interpretative paradigm carrying out interviews to the experts of this topics and analyzing the information from The Grounded Theory of Glaser \& Strauss. Among the main findings, one finds that a methodological level evaluation modalities stands out as the psychosocial and psychological-experts look; and that the attachment and empathy come up as the main elements to appraise the referents. As well, as to the techniques and instruments used were the most used the Semi-constructed Interview and the Rorschach Test. On a theory level the perspective used are the systemic, ecologist and the clinic-expert approaches. On the other hand, the professionals offer a mayor valuation to the experience over the theory formation, referring as notable personality characteristics the empathy, for a better developed process. Finally, it is necessary to indicate that the main weakness of the process was the lack of standardization parameters, methodological as theoretical standardization, to understand and evaluate the parental skills, task that at present is done according to the assessors, bordering the objectivity; and impeding making decisions regarding the habilitation and immobilizations of a referent, affecting the principle of Superior Interest of Child.

KEY WORDS: Parental Skills, Evaluation, Family Right.

Corrrespondencia: Debe ser dirigida a Octavio Astudillo.

Email:..0sam85@hotmail.com; 2. ivy_g_@hotmail.com; 3.c_retamales@live.cl; 4.mayrojas@ucn.cl; 5. wsarria@hotmail.com. 


\section{INTRODUCCION}

El censo efectuado por el Instituto Nacional de Estadísticas [INE] en el año 2002, evidencia el incremento en Chile de los hogares monoparentales frente a la conformación nuclear biparental, evidenciando el cambio experimentado por los hogares chilenos en su estructura familiar, condiciéndose con el aumento del número de disoluciones matrimoniales durante los últimos años. Por otra parte se ha observado un aumento de las problemáticas asociadas a este cambio, afectando principalmente a los/as niños/as y adolescentes, por cuanto éstos tienden a ser más vulnerables a negligencias, maltratos $\mathrm{y}$ abusos.

Frente a esta desprotección legal de los/as niños/as, Chile empieza a crear leyes que buscan resguardar el interés superior de éstos, sin embargo, las leyes sobre derecho de menores no dieron respuesta a los problemas generados a consecuencia de conflictos familiares. Ante esto, en el año 2005, mediante la ley 19.968 entran en vigencia los Tribunales de Familia, los cuales tiene como uno de sus objetivos "el conocer y resolver las causas relacionadas al derecho de cuidado personal de los niños, niñas o adolescentes; y los derechos $y$ deberes del padre o madre que no tenga el cuidado personal del hijo, entre otras materias que también derivan de las relaciones de familia” (García, Sir \& Telchi, 2007, p. 5).

Con lo anterior se da énfasis en el derecho de los/as niños/as a ser cuidados, más que el derecho-deber de los padres de asistirlos.

Respecto a esto la ley 19.585 del código civil, establece, que en el caso de separación, si no existe acuerdo entre los padres sobre quien detentará el cuidado del/la niño/a será necesario recurrir a los Tribunales de Familia para solicitar el derecho al cuidado personal de éstos. Ante estos casos, la ley 19.968 en su artículo 45 señala que el juez, de considerarlo pertinente, puede solicitar un informe pericial a profesionales expertos, cuyo objetivo es evaluar habilidades parentales de ambos padres, con el fin de determinar cuál resulta más idóneo para que se le otorguen los cuidados personales de los/as hijos/as, o en otros casos, evaluar a otros referentes familiares, si los padres se encuentran inhabilitados física y/o mentalmente.
Cabe destacar que lo anterior también es aplicado a los casos de vulneración de derecho de los niños/as y adolescentes.

Consecuentemente con lo expuesto, las habilidades parentales que debe tener el/la referente que obtenga el cuidado personal del/la niño/a, no se encuentran establecidas al alero de un consenso único por el cual se pueda establecer dimensiones específicas para ello, por cuanto éstas dependerán del enfoque teórico al que se adscriba el/la evaluador/a, de la situación sociopolítica en la que se enmarque, entre otros. Sin embargo, a la luz de diferentes estudios, mayoritariamente internacionales, ha sido posible identificar algunos factores comunes que permiten comprender de mejor forma este fenómeno tan complejo de operacionalizar. Entre estos estudios se puede señalar a Clausen (1968, citado en Otto \& Eden, 2000) quien fue uno de los primeros autores en referirse al tema; además de otros autores como Chasen \& Gruenbaum (1981, citado en García et al., 2007), y Barnard \& Jenson (1984, citado en García et al., 2007), quienes realizaron variados aportes respecto a las dimensiones que componen el concepto de habilidades parentales. Así también, existen autores que plantean diferencias entre habilidades $y$ competencias parentales, dificultando aún más establecer criterios comunes. Debido a lo anterior, en esta investigación se utilizará lo señalado por tres fuentes; Jorge Barudy quien es uno de los autores que se aproxima al contexto nacional, la Asociación Chilena de Psicología Jurídica y el Servicio Nacional de Menores [SENAME], estando estos dos insertos en el mismo contexto.

De acuerdo a Barudy (s.f.) las competencias parentales "son una forma semántica de referirse a las capacidades prácticas que tienen los padres para cuidar, proteger y educar a sus hijos, asegurándoles un desarrollo suficientemente sano" (p. 3). Además, señala que dichas competencias parentales, se encuentran dentro de lo que se denomina parentalidad social definida por SENAME (2007) como "la capacidad para responder a las necesidades fundamentales de los hijos como lo son la alimentación, cuidados corporales, protección, entre otras. Importante de mencionar es que éstas necesidades son evolutivas y múltiples por lo que los padres deben adaptarse a dichas necesidades" (p. 3). 
Es en este punto, donde Barudy incluye las habilidades parentales, refiriéndose a ellas como un componente evaluable de la parentalidad.

A nivel nacional, la Asociación Chilena de Psicología Jurídica y Forense, establece que las competencias parentales "incluyen diversas capacidades individuales de los padres para facilitar el desarrollo físico, afectivo, intelectual y social de sus hijos. Entre tales competencias destacan la capacidad para generar un vínculo de apego seguro con los hijos; la capacidad y el interés por estimular el desarrollo psicológico y social a través de la conversación y el juego, entre otras actividades; la capacidad para ofrecer medios adecuados y oportunos para la satisfacción de necesidades de los hijos; la capacidad para supervisar y controlar el comportamiento de los hijos mediante la imposición de normas claras y consistentes orientadas a su protección; etc." (s.f., p. 1).

Por último, SENAME, utiliza la misma definición empleada por Barudy para referirse a las competencias parentales, sin embargo, distingue entre éstas y las habilidades parentales, definiendo estas últimas como: "la plasticidad de las madres y los padres, que les permite dar una respuesta adecuada $y$ pertinente a las necesidades de sus hijos de una forma singular, $y$ de acuerdo con sus fases de desarrollo" (SENAME, 2007, p. 7). Teniendo en cuenta que "dicha plasticidad se basa en las experiencias de vida en un contexto social adecuado, donde se produzcan los procesos adaptativos y el apoyo social necesario para hacer frente a situaciones de estrés con las que se enfrenta la familia" (SENAME, 2007, p.7). Junto con lo anterior, SENAME considera que un/a padre/madre competente tiene que poseer cuatro características fundamentales, tales como "capacidad de apegarse a los hijos/as, la empatía, los modelos de crianza y la capacidad de participar en redes sociales y de utilizar los recursos comunitarios" (SENAME, 2007, p. 7). Respecto a lo anterior es importante señalar que si bien, a nivel teórico se plantean tanto diferencias como similitudes entre ambos conceptos, para efectos de la presente investigación se considerará el término habilidades parentales, ya que desde Tribunales de Familia se solicita la evaluación de éstas en los diferentes oficios.
Las habilidades parentales han estado sujetas a diferentes tipos de estudios que ayudan a comprender su relación con la crianza de niños/as, estando entre estos los que enfatizan el desarrollo de instrumentos específicos para la evaluación de las habilidades parentales, siendo uno de los instrumentos más usados en Norteamérica, el Ackerman-Shoendorf Scale of Parent Evaluation of Custody (ASPECT), escala que evalúa comparativamente a la madre y al padre (García et al., 2007). En relación al ASPECT, la primera aproximación en Chile consistió en un proceso de adaptación semántica en el año 2007 por García et al., no siendo estandarizado para la población chilena, imposibilitando su actual utilización.

Como resultado de estas investigaciones dentro del contexto internacional se han establecido otras formas de evaluación. Tal es el caso de Norteamérica, donde se toman en cuenta diferentes factores tales como, los deseos de los padres del/la niño/a sobre su custodia'; los deseos del/la niño/a sobre quien detente la custodia; la interacción e interrelación entre el/la niño/a y cualquier persona que pueda afectar significativamente sus mejores intereses; el ajuste del/la niño/a a su entorno y la salud física y mental de todos los individuos implicados (Rodríguez \& Avila, 1999); además se considera el contexto cultural de cada familia evaluando a sus miembros considerando su interacción y relación. (Reebye, s.f.). Por otra parte, en España, Rodríguez \& Avila (1999), señalan que el método de evaluación de custodia es "fundamentalmente observacional (directa e indirecta) utilizando las entrevistas semi-estructuradas como un recurso básico para la recogida de datos y la inclusión de instrumentos de evaluación forense" (p. 123). En este sentido Bronchal (s.f.) señala como crítica a este procedimiento, la inexistencia de criterios decisorios para la evaluación, generando una inapropiada metodología para este proceso, cuestionando básicamente el uso de instrumentos psicológicos utilizados clínicamente.

En el contexto latinoamericano, con respecto al proceso evaluativo, Barudy \& Dantagnan (2005) proponen un método basado en la

\footnotetext{
1 Cabe señalar que en el contexto internacional se utiliza el término Custodia, mientras que en el contexto nacional se emplea, desde el año 2005, el término Cuidado Personal.
} 
observación participante, el cual consta de 5 niveles de evaluación: "las características individuales de los padres; las evaluación de las características de las relaciones sociales de los padres con su comunidad; la evaluación de la capacidad de los padres de solicitar $y$ beneficiarse de organismos y profesionales; la valoración de la calidad de las relaciones de los padres con sus hijos; y la observación y evaluación de las habilidades parentales actuales". (pp. 11-12).

En Chile, Zúñiga (2003), antes de la instauración de los nuevos Tribunales de Familia, planteaba que la evaluación debía realizarse a través de entrevistas semiestructuradas a ambos padres, a los/as niños/as y entrevistas a los padres e hijos/as en conjunto; además de la aplicación de pruebas psicológicas. Posteriormente, con la nueva justicia de familia, fue necesario revisar los procedimientos hasta entonces utilizados; sin embargo, los escasos estudios orientados a explorar los procesos de evaluación de habilidades parentales pusieron de manifiesto la existencia de distintas metodologías utilizadas y las dificultades que esta práctica podría generar en las causas de cuidado personal. Así, en cuanto a los centros de diagnósticos dependientes de SENAME, correspondientes a la IV, VI y Región Metropolitana, se señala, "que en el $41 \%$ de los casos cuentan con una pauta predefinida, los que en su totalidad expresaron que corresponde a una pauta establecida por el mismo centro de atención" (García et al., 2007, p. 38). Además de esto, los investigadores también establecen que en general se evalúa a todo el grupo familiar, pero existen instituciones donde el proceso de evaluación de habilidades parentales sólo se realiza con una evaluación del/la niño/a. Respecto a las dimensiones consideradas, concluyen que el vínculo aparece como más relevante; luego se encuentran las habilidades y la actitud de los padres, la interacción padre-madre e hijo/a, psicopatología, personalidad y capacidad intelectual y finalmente el nivel socioeconómico.

Actualmente en Chile la responsabilidad de realizar las evaluaciones de habilidades parentales por encargo de los Tribunales de Familia, recae en las direcciones regionales de SENAME a través de sus unidades especializadas; en los distintos Centros de
Diagnóstico dependientes de SENAME, como también en el Servicio Médico Legal [SML].

Consecuentemente con lo anterior, a pesar de que en Chile existen instituciones abocadas específicamente a esta labor, la evaluación diagnóstica no se encuentra debidamente estipulada en lineamientos institucionales. En el caso de SENAME (2007), plantea que "para el caso de evaluaciones de competencias parentales se recomienda la comprensión y el estudio de lo que se espera de los padres o adultos significativos para el proceso de crianza y cuidados de los niños y las niñas. Frente a la evaluación de presencia de indicadores clínicos de relevancia se espera que los adultos cuenten con una evaluación de capacidades cognitivas y estructura de personalidad" (SENAME, p.15).

Esto genera que la metodología y los criterios para evaluar habilidades parentales, en los casos de cuidado personal, quede a elección de los distintos centros de diagnóstico dependientes de SENAME, los cuales pueden adoptar diferentes modalidades para evaluar el mismo fenómeno. Lo anterior queda señalado en las bases técnicas específicas de la línea diagnóstica de SENAME, correspondiente al año 2007, la cual señala que "Ios enfoques de trabajo deberán ser definidos por el equipo ejecutor, el cual tendrá la libertad para delimitar, planificar y desplegar las estrategias necesarias para gestionar el programa, implementando una acción coherente con el desarrollo de los objetivos del mismo" (SENAME, 2007, p. 7).

Sin embargo, lo anterior afectaría los resultados de las evaluaciones, por cuanto podrían variar según los criterios establecidos para el diagnóstico, dificultando la toma de decisiones con respecto al beneficio del/la niño/a. De esta manera, la heterogeneidad de criterios teóricos y metodológicos generaría discrepancias a la hora de triangular la información, tanto entre profesionales como entre instituciones, los que basan sus decisiones en este tipo de diagnóstico.

Por lo expuesto, al no existir lineamientos comunes y consensuados por las instituciones involucradas, resulta necesario sistematizar y estructurar este procedimiento de evaluación, en un formato común ayudando a homogenizar el proceso, permitiendo responder de manera prolija a los requerimientos de Tribunales de 
Familia en las causas de cuidado personal, aportando de manera significativa al desarrollo de la psicología jurídica, en el ámbito del derecho de familia, dado los escasos estudios a nivel nacional y regional. Esto constituiría un aporte para evitar discrepancias, dobles evaluaciones, re-victimización, variados formatos de informe, entre otros; dificultades que actualmente yacen en el sistema de evaluación de habilidades parentales, ya que según Bronchal (s.f.), en ocasiones la intervención psicológica puede ser, "un factor generador de más problemas de 10 que pretende resolver"(p. 5). Sin embargo, como primer paso resulta necesario conocer el procedimiento de evaluación tomando en consideración los distintos aportes de los profesionales de las ciencias sociales que trabajan en el ámbito del derecho de familia, debido a que éstos intervienen directamente en el proceso de evaluación; en el que una metodología de tipo cualitativa permitiría obtener una visión directa y especifica respecto al procedimiento llevado a cabo actualmente. Esto podría dar pie a futuras investigaciones para generar criterios y metodologías comunes entre las distintas instituciones que intervienen en el proceso de diagnóstico y/o evaluación de habilidades parentales.

Es por estos motivos que el presente estudio pretende, en primer lugar conocer, con mayor detalle, los procesos de evaluación de habilidades parentales propuestos por las instituciones estudiadas, con el fin de poder señalar las fortalezas y debilidades de éstas, desde la perspectiva de los/as profesionales relacionados con dichas evaluaciones. Sintetizando lo anterior, se formula la siguiente pregunta de investigación ¿Cómo es el proceso y cuáles son los criterios de evaluación diagnóstica de habilidades parentales, utilizados por los profesionales pertenecientes al ámbito del derecho de familia de la ciudad de Antofagasta?

Con lo anterior, se propone como objetivo general de investigación: Describir el proceso de evaluación de habilidades parentales, llevado a cabo por los/las profesionales pertenecientes al ámbito del derecho de familia de la ciudad de Antofagasta.

Para lograr el objetivo antes mencionado, se proponen como objetivos específicos: 1. Describir la metodología de trabajo utilizada en la evaluación de habilidades parentales; 2. Identificar los enfoques teóricos a los que se adscriben los/as profesionales para llevar a cabo el proceso de evaluación de habilidades parentales; 3. Identificar las principales fortalezas y debilidades del proceso de evaluación de habilidades parentales; 4. Describir la formación y experiencia de los/as profesionales, respecto a la labor de evaluación de habilidades parentales; y finalmente, 5 . Conocer las sugerencias de los/as profesionales para mejorar el proceso de evaluación de habilidades.

\section{MÉTODO}

La metodología usada para llevar a cabo la investigación es de tipo cualitativa, ya que permite acceder a la visión subjetiva, experiencias y vivencias de los/as profesionales vinculados al proceso de evaluación de habilidades parentales.

\section{Participantes}

Para la presente investigación se consideró un muestreo intencional (Ruiz, 2003), permitiendo seleccionar a los sujetos de estudio en base a características deseadas e idóneas. De esta manera la unidad de análisis estuvo compuesta por un total de siete profesionales de la ciudad de Antofagasta, pertenecientes al sistema psicosocio-jurídico ${ }^{2}$, de los cuales seis corresponden al sexo femenino y uno al sexo masculino, con un rango etario entre 29 y 39 años. Además se consideró como otros criterios de inclusión el que realicen o hayan realizado evaluaciones de habilidades parentales a partir del año 2005, año en que entraron en vigencia los Tribunales de Familia; y que tuvieran al menos un año de experiencia en este tipo de evaluaciones, tal como se señala en la Tabla $N^{\circ} 1$.

\section{Procedimiento General}

Para llevar a cabo la presente investigación, se trabajó en una serie de etapas concadenadas que se detallan a continuación: a) Revisión bibliográfica exhaustiva acerca de las habilidades parentales, desde su concepción

2 Profesionales Psicólogos y Asistentes Sociales que trabajan en el sistema judicial chileno. Como por ejemplo Tribunal de Familia, Tribunal Oral en lo Penal, etc. 
hasta procesos de evaluación, permitiendo el desarrollo de criterios necesarios para sentar las bases del proceso investigativo, b) Confección de Eje y Guión Temático, necesario para conducir la recolección de datos a través de la entrevista, c) Contacto a través de redes, para luego formalizar la instancia por medio de una invitación escrita y posterior coordinación con los/as profesionales para establecer los aspectos formales de los encuentros, d) Una vez contactados los/as profesionales, se efectuaron las entrevistas en forma individual y en las dependencias laborales de los/as operadores/as. Cabe señalar que en algunas oportunidades la obtención de información se efectuó en dos sesiones. Se utilizó un consentimiento informado, el cual explicitó el objetivo de la investigación, así como también los temas éticos relacionados con el anonimato y confidencialidad en la entrega de la información. e) Los criterios de validación utilizados son la Credibilidad, a través de la triangulación de datos, específicamente de actores y expertos; la Dependibilidad, debido a que esta investigación estuvo sometida a un control externo, determinando la idoneidad del proceso realizado (Ramírez \& Villarroel, 2006) y finalmente se logró una aproximación a la Saturación, una vez que la información se reiteró en los entrevistados. f) De forma paralela se efectuaron las transcripciones de cada entrevista, procediendo al análisis de información, ejecutando en forma sucesiva la codificación abierta, axial y una aproximación a la codificación selectiva.

\section{Procedimiento de Recolección y Análisis de la Información}

Para recabar la información necesaria se utilizó como técnica de recolección de información la entrevista a expertos. Esta entrevista se confeccionó mediante el uso de un guión temático previamente estructurado basado en tres ejes temáticos: metodología y técnicas utilizadas en la evaluación de habilidades parentales; enfoque teórico utilizado en la evaluación de habilidades parentales; y formación y experiencia de los/as profesionales evaluadores/as de habilidades parentales, los cuales fueron elaborados de acuerdo con los objetivos de la investigación. Cabe señalar que para la recolección de la información se usaron dispositivos digitales de captación de voz, siendo autorizado a través del consentimiento informado, logrando información fidedigna al momento de transcribir y analizar.

El análisis de los datos obtenidos por medio de las entrevistas, se realizó a través de los planteamientos de la Teoría Fundamentada (Grounded Theory) de Glaser \& Strauss. Se construyó un esquema explicativo e integrado, a través de la visión de los/as profesionales, sobre el proceso evaluativo identificando en los discursos temas comunes y disidentes entre ellos. Luego de la transcripción de la información obtenida, se realizó el proceso de codificación abierta, con el fin de identificar las categorías iniciales de la investigación; posteriormente se llevó a cabo la codificación axial, analizando cada categoría obtenida; para finalmente realizar la aproximación a la codificación selectiva, por medio de la integración de los datos vislumbrados en la presente investigación (Ramírez \& Villarroel, 2006).

\section{RESULTADOS}

En lo que respecta a la evaluación de habilidades parentales, formalmente no existen parámetros estandarizados para permitir una evaluación prolija a lo largo de todo el país, dejando en manos de diferentes organismos y profesionales la elección tanto teórica como metodológica en este tipo de evaluación, llevando a que en muchos aspectos coincidan, pero a la vez difieran en otros puntos relevantes.

El análisis descriptivo de la información obtenida, permite entregar los resultados en siete categorías, supeditadas a los objetivos específicos propuestos en esta investigación. Estas categorías describen tanto la metodología utilizada en la evaluación de habilidades parentales, el enfoque al cual se adscriben los/as profesionales evaluadores/as, las fortalezas y las debilidades del proceso de evaluación llevado a cabo actualmente, las sugerencias para mejorar el proceso de evaluación, y finalmente, la perspectiva personal de los/as profesionales frente al concepto de habilidades parentales.

\section{Proceso de Evaluación y Metodología}

El proceso de evaluación de habilidades parentales comienza con la solicitud emanada 
desde Tribunal de Familia, teniendo presente en primera instancia dos grandes aspectos:

\section{Estrategia y Procedimiento.}

La Estrategia contempla el número y tipo de sesiones y el trabajo interdisciplinario. De esta manera, el número de Sesiones, fluctúa entre una y dos, encontrando profesionales que evalúan, en una sesión, consistente en un peritaje psicológico, enfocado a obtener las características significativas de personalidad del referente evaluado. A diferencia de otros profesionales quienes evalúan en dos sesiones, orientadas a la realización de una evaluación psicosocial, donde en primera instancia se efectúa una visita domiciliaria dirigida a conocer el contexto socioeconómico y familiar, labor realizada por un/una Asistente Social. En segunda instancia se realiza una entrevista psicosocial orientada a indagar en antecedentes familiares y sociales, como también en características psicológicas del referente evaluado, tarea realizada por un/una Asistente Social y un/una Psicólogo/a respectivamente. Cabe señalar que cada instancia de evaluación tiene una duración promedio de dos horas. El lugar donde se realizan las sesiones de evaluación, son las dependencias de las instituciones donde trabajan los/as profesionales, como también en el caso de la evaluación psicosocial, que contempla una visita domiciliaria realizada en el hogar del referente.

En cuanto al Trabajo Interdisciplinario, éste alude al análisis que se realiza entre las líneas intervinientes, estableciéndose un análisis psicosocial realizado por un/a Psicólogo/a y un/una Asistente Social cuya finalidad es conocer el contexto de los evaluados, un análisis psicopatológico realizado por una Psicóloga y un Psiquiatra, donde la primera puede solicitar derivación para el segundo con el fin de descartar sospecha de patología, y por último un análisis psicosocialjurídico donde una Psicóloga y la Asistente Social son asesorados por un/a Abogado/a.

"Nosotros tenemos una
metodología, que parte con la
aplicación de las pruebas psicológicas
mencionadas, luego se pasa a una
etapa de entrevista conjunta con la
asistente social, una entrevista
psicosocial en profundidad [...]

Posterior a eso viene el análisis de la dupla en relación a los antecedentes recogidos". (E1)

El segundo aspecto es el Procedimiento, el cual contempla tres fases; la primera fase Análisis Preliminar de los Antecedentes, que conlleva las fuentes de información y las dimensiones a evaluar. Las fuentes de información pueden ser directas y tienen que ver con los referentes a evaluar que son solicitados por Tribunal o solicitados por las propias instituciones si fuese necesario. Mientras que las indirectas tienen que ver con las carpetas de información que llegan desde Tribunal o que pueden provenir desde otras instituciones.

"Lo que hacemos nosotros es ir revisando todo lo que es material que tengamos, todo lo que es material escrito por así decirlo, los informes, quienes visitaron, la cantidad de las visitas, la calidad, la vinculación que el niño haya tenido con la familia, el motivo por el cual se generó la medida de protección [...] cuando uno ya maneja la causa, es cuando empezamos la entrevista". (E5)

Las dimensiones a evaluar, aluden a las diferentes áreas a valorar en el referente, entre las que se encuentran: Familiar, donde se revisa la historia vital y los conflictos relacionales; Salud Mental, donde se evalúa la estructura de personalidad, las posibles patologías y el consumo de sustancias; Cognitiva, donde se evalúa el coeficiente intelectual (C.I.); Afectiva, donde se revisa el nivel de apego y empatía; Conductual, donde se ven los estilos de crianza y la plasticidad parental; y por último, Social, donde se revisa el uso de redes familiares y comunitarias y el nivel socioeconómico.

"[...] finalmente se logró tener un apartado, particularmente de habilidades parentales, y ahi se definen 6 puntos... el primero avocado a la historia personal, [...] orientado al recurso parental, que tiene que ver con el antecedente familiar del referente; la capacidad de empatizar con la vivencia del niño, niña o adolescente... que en este caso pretende asumir su cuidado; el nivel 
de apego que mantiene con él o ella... o ellos; tiene que ver con la capacidad de establecer modelos de crianza [...] si se configura como un referente de autoridad; tiene que ver con la capacidad de adherirse a las redes sociales, de vincularse a las redes sociales, de recibir asistencia entorno a esta red... y en el fondo lograr que su situación como referente con el niño incorporado sea visualizada socialmente; y finalmente, con los antecedentes de salud mental...." (E2)

La segunda fase comprende la Evaluación del Nivel de Habilidades Parentales, que contempla la obtención del relato y aplicación de técnicas e instrumentos. En la obtención del relato se encuentran las fuentes de registro que pueden ser escritas o audio. De acuerdo a lo señalado por los/as profesionales, las técnicas utilizadas son: entrevista semi-estructurada psicosocial, entrevista semi-estructurada clínicopericial y por otra parte la observación directa, que contempla la visita domiciliaria, la escala de observación de apego, basados en los Criterios Care-Index; como finalmente la utilización del genograma, como técnica complementaria. Ahora bien, los instrumentos aplicados se pueden clasificar en tres categorías: test proyectivos aperceptivos, entre los que destaca el Test de Rorschach como el instrumento común entre Psicólogos/as; pruebas psicométricas como el WAIS y 16 PF; y test proyectivos gráficos, destacando el Test de Persona Bajo la Lluvia, como el más utilizado.

“[...] En el área afectiva, el tipo vivencial, [...] la capacidad para poder establecer relaciones, la capacidad empática, si hay algunos indicadores de egocentrismo y también el tipo de vínculo que se establece que se puede también ver a través del Test de Rorschach; también el tema de la vida impulsiva, ver como controla los impulsos, si es capaz de controlarlo o si más bien tiende a gratificar no más el tema de los impulsos inmediatos, desconsiderando el contexto...". (E6)

La última fase corresponde a la Determinación del Nivel de Habilidad Parental, que contempla cuatro niveles: análisis de la información, toma de decisiones, redacción del informe y declaración en Tribunal de Familia. El análisis de la información se realiza de acuerdo a lo recabado por las técnicas e instrumentos a nivel cualitativo y cuantitativo. La toma de decisiones contempla las dimensiones evaluadas que se dividen en satisfactorias, regulares e insatisfactorias. Lo satisfactorio se refiere a que las condiciones que debe reunir el/la referente evaluado/a para estar habilitado/a como cuidador/a del/la niño/a; lo regular se relaciona con los elementos que podrían imposibilitar al/la referente en su rol como cuidador/a, pero son elementos que pueden ser modificados, catalogándolo como habilitable; y por último insatisfactorio en el que el/la referente no reúne las condiciones 0 existen elementos inmodificables que impidan su desempeño como cuidador/a, tales como un bajo C.I. o un trastorno psiquiátrico grave, catalogándolo como un/a referente inhabilitado/a.

"Primero la persona que está habilitada tiene que reunir ciertas competencias, tiene que tener ciertas características como por ejemplo, ser capaz de ver las necesidades del menor, ser capaz de evaluar riesgos [...] La persona que esta inhabilitada pero con posibilidad de habilitarse [...] son las personas que tienen actualmente una patología que impide que ejecuten bien su rol, pero no tienen que ver con una cosa estructural, no tiene que ver con algo que sea muy difícil de modificar o que no se pueda modificar [...]. Y está la otra parte que tiene que ver con la inhabilidad propiamente tal, desde el punto de vista de nosotros, que tiene que ver con factores que tienen que ver con negligencias". (E3)

El tercer nivel consiste en la redacción del informe, estableciéndose dos tipos, psicológico y psicosocial. El informe psicológico, contempla en su estructura, la historia vital, pruebas aplicadas y conclusión el cual incluye ámbito parental, conclusiones generales y recomendaciones. En cambio los informes de tipo psicosocial contempla dos tipos de estructura, según el/la profesional que evalúa; el primer tipo considera identificación, antecedentes relevantes, aplicación de instrumentos, antecedentes sociales, antecedentes psicosocial, habilidades 
parentales, conclusiones y sugerencias; mientras que el segundo tipo contempla individualización, identificación del/la niño/a, motivo de evaluación, procedimientos, desempeño del rol, vinculación con el/la niño/a, antecedentes habitacionales y económicos, antecedentes penales, antecedentes psicológicos y conclusiones.

"tiene la parte de la de la historia vital del sujeto, las pruebas aplicadas y después viene el tema de las conclusiones y dentro de las conclusiones hay un pequeño párrafo que es el tema del ámbito parental, del como la persona se desenvuelve dentro o qué cosas están afectando el ejercicio de su rol. $Y$ después viene la conclusión general que es si la persona está habilitada o inhabilitada y después las recomendaciones que sean pertinentes para el caso". (E3)

Por último esta fase y el proceso de evaluación finalizan con la declaración en Tribunal de Familia, por parte de los/las evaluadores/as, en los casos que este organismo estime conveniente.

\section{Perspectiva Teórica}

Relacionado con los aspectos teóricos de la evaluación, se abren tres líneas de análisis con respecto al Enfoque Teórico. La primera corresponde al enfoque teórico al que los/as profesionales se adscriben de manera Personal, los cuales corresponden a los enfoques sistémico, ecológico, psicoanalista y humanista, existiendo un mayor número de profesionales adscritos al enfoque sistémico.

"Generalmente uso el sistémico, que involucra todo, como van influyendo todos los aspectos. Porque tu vas viendo como se utilizan las redes sociales, como están las mismas redes familiares, como intervienen [...]". (E5)

"[...] la visión que tengo para lograr comprender la problemática tiene que ver particularmente con un enfoque ecológico, a que me refiero con esto, yo no puedo lograr comprender la situación puntual solamente desde las personas que yo evalúo sin atender a la realidad contextual, relacional, sociocultural [...]". (E2)

"Es que, por ejemplo, yo tengo un enfoque más psicoanalista... [...]". (E4)

"[...] mi postura clínica, es humanista, humanista experiencial, y que me favorece mucho en el tema del peritaje porque me da una buena llegada con el paciente [...]". (E3)

La segunda línea se relaciona con el enfoque teórico al que se adscriben como Evaluadores/as de Habilidades Parentales, siendo en la mayoría de los casos coherente con su enfoque personal, evidenciándose concordancia en la obtención y análisis de la información, sin embargo, esto difiere en algunos casos, donde el enfoque utilizado en la evaluación no corresponde al utilizado personalmente. Dentro de esta línea se pueden identificar los siguientes enfoques teóricos: ecológico, en el que destacan autores como Barudy y Bronfenbrenner como referentes significativos; sistémico, donde Duvall se constituye como referente dentro del análisis; y finalmente, clínico-pericial; que según lo referido por la entrevistada, es básicamente el enfoque clínico contextualizado en el ámbito jurídico, tanto en la recolección, análisis y conclusiones de los datos obtenidos. La última línea de análisis, corresponde al enfoque teórico al que se adscriben las Instituciones en las que trabajan los/as evaluadores/as, relacionándose de manera directa con las discordancias expuestas anteriormente, debido a la adaptación a los marcos reguladores institucionales por parte de los/as profesionales; en este sentido destacan como enfoques institucionales: el ecológico, supeditado a Barudy; sistémico y clínico-pericial.

"A ver, para evaluar las habilidades parentales [...], como desde un enfoque más sistémico, más ecológico, más integral de la persona, independiente de que uno tenga otra visión, otra perspectiva de las cosas, pero siento que en este contexto, en este caso, lo que podría dar respuesta [...]". (E4) 


\section{Recursos Profesionales y Personales}

Dentro de la evaluación de las habilidades parentales, la figura del/la profesional emerge como un pilar fundamental dentro del proceso, ante lo cual el Nivel de Preparación es clave para realizar un trabajo prolijo y especifico. De acuerdo a los/as entrevistados/as, la evaluación, desde el punto de vista del/la evaluador/a, obedece a dos aspectos fundamentales: la Experiencia y la Formación.

La Experiencia, abarca el desenvolvimiento teórico-práctico que han tenido los/as profesionales tras su formación de pre-grado en el ámbito laboral, dividiéndose en áreas de trabajo, evaluación de habilidades parentales y capacitación profesional. En cuanto a las áreas de trabajo, los/as profesionales se han desempeñado, tanto con adultos como con niños/as y adolescentes en áreas como rehabilitación de drogas, vulneración de derecho de menores, tratando los temas de explotación sexual en niños/as; psicología legista, realizando evaluaciones psicológicas de carácter pericial; y finalmente, socialcomunitaria. El segundo elemento considerado es la evaluación de habilidades parentales, donde los/as profesionales llevan desempeñándose en promedio tres años, con una cantidad de informes elaborados que fluctúa entre los 200 y 800 . Finalmente, el tercer elemento considerado es la capacitación profesional, que incluye cursos, capacitaciones y seminarios considerados escasos.

"Yo me titulé como yo te dije en el año 2000, yo del 99 vengo trabajando en el "Hogar de Cristo", eso es como experiencia laboral en general, desde el año 2004 que fue cuando entró en vigencia, en octubre de ese año, la Ley de Tribunales de Familia, nosotros empezamos a desarrollar estos informes". (E5)

Por otra parte, la Formación, considera los factores personales, formación de pre-grado y post-grado. De esta manera, se establece que los factores personales pueden influenciar la evaluación de dos formas, actuando como facilitador y/o obstaculizador. Dentro de los facilitadores se encuentran principios éticos como confidencialidad, donde la información sólo es usada para fines judiciales; interés superior del niño, donde los/as evaluadores/as

consideran como principal convicción velar por el bienestar del/la niño/a al momento del análisis; capacidad de inhabilitación, referida a eximirse de realizar el proceso en casos donde exista relación con los/as referentes a evaluar; y finalmente, no sesgar la evaluación, debido a que podría generar prejuicios al momento de elaborar las conclusiones. Otro facilitador considerado como relevante por algunos/as profesionales son las características de personalidad encontrándose la empatía como una forma de establecer una comunicación eficaz con el/la referente a evaluar. Otra característica es la atención sostenida relacionada con la capacidad para atender a toda la información entregada por el/la referente durante la entrevista; y la capacidad de análisis en el transcurso de la propia entrevista. Sin embargo, para un grupo mínimo de profesionales, estas características no son relevantes dentro del proceso. En cuanto a los obstaculizadores se encuentra la hipersensibilidad donde el/la evaluador/a puede ser afectado emocionalmente por el/la referente comprometiendo su objetividad, así como los conflictos no resueltos por parte del/la evaluador/a.

\begin{abstract}
"[...]se requiere que la persona sea muy empática, porque recordemos que como es un ámbito clínico, por lo tanto la persona no viene voluntariamente acá, viene además dentro de un proceso donde muchas veces están siendo acusados de delitos, entonces lo básico, es ser muy empático". (E3)
\end{abstract}

En cuanto a la Formación de Pre-grado, los/as profesionales tuvieron formación académica en instituciones como: Universidad de Antofagasta, Universidad Católica del Norte, Universidad de Tarapacá y Universidad José Santos Ossa, (actualmente Universidad del Mar). Con respecto a la Formación de Post-grado, uno de los/las profesionales tiene un post-título en pericias forenses mientras el resto tiene una formación post-grado en otras materias que no consideran relevantes para la evaluación de habilidades parentales.

\section{Fortalezas}

Desde la mirada de los/as profesionales, es posible rescatar que en el proceso evaluativo 
actual de habilidades parentales las Fortalezas se encuentran dentro de tres áreas, concernientes a Metodología, Perspectiva Teórica y Recursos Profesionales.

Dentro de la Metodología utilizada, los/as profesionales refieren en primer lugar que el trabajo psicosocial realizado, específicamente relacionado a la participación de la dupla psicosocial en la visita domiciliaria, percibida como una de las principales fortalezas a nivel metodológico, evidenciándose por casi la totalidad de los/as entrevistados. En segundo lugar, señalan como fortaleza la validación externa que existe desde los/as operadores/as del Tribunal de Familia, principalmente de los/as Magistrados/as, con respecto a la redacción de los informes de una institución en particular, hecho que se evidencia en la alta consideración que existe respecto de sus resultados y sugerencias para dictaminar en las causas asociadas a esta materia. En tercer lugar, algunos/as profesionales identifican como fortaleza el carácter integral establecido para evaluar, haciendo referencia a la dirección que ha tomado el proceso evaluativo realizado por algunos/as profesionales, considerando el contexto completo donde está inmerso el/la niño/a. Finalmente, se encuentran los aspectos a evaluar, donde uno de los/as entrevistados refiere como fortaleza considerar la estructura de personalidad y C.I. de los/as referentes.

Las fortalezas correspondientes a la Perspectiva Teórica, están referidas a la utilización del enfoque ecológico y el enfoque humanista experiencial, los que son muy valorados por los/as profesionales que los utilizan. En relación al primero, la fortaleza recae en la mirada global del sistema que éste le entrega, como también, en la visión del/la niño/a dentro de un contexto socio-familiar, lo que ayuda a identificar diferentes dinámicas que surgen entre el/la niño/a, el/la referente y el contexto donde se desenvuelven. $Y$ por otro lado, el segundo aspecto se refiere a la relación con el/la evaluado/a, la que se establece de mejor forma con la utilización de herramientas de este enfoque, traduciéndose en una apertura del/la referente durante la entrevista.

"Yo pienso que la principal fortaleza, es que se visualiza al niño dentro de un contexto, no hay niño como un ser autónomo, individual. $Y$ también el otro punto a favor es que hay una visión integrada, psicosocial acerca de la realidad de la familia, de la problemática, es un diagnostico integral y donde el niño que es sujeto de nuestra atención esta visualizado en un contexto socio familiar". (E1)

Por último, dentro de los Recursos Profesionales, se evidencian como fortaleza la experiencia evaluativa de los/as profesionales de esta área; la formación, considerándose en este punto el conocimiento y manejo de las técnicas de evaluación y la actualización de contenidos, lo que es posible mediante la capacitación de los/as profesionales. Cabe destacar, que los/as profesionales entregan una mayor valoración a la experiencia que a la formación en la realización de este tipo de evaluación, constituyéndose como una fortaleza significativa. Finalmente emergen como fortalezas las características personales, específicamente en lo que se refiere a poseer capacidad de atención, capacidad de análisis y rigurosidad durante la entrevista y el proceso evaluativo.

"Yo creo que es súper importante la formación, pero la experiencia te da esa tranquilidad de cuando tú estás haciendo las cosas que realmente estas convencido de que efectivamente es así". (E6)

\section{Debilidades}

Así como los/as profesionales señalan la existencia de fortalezas en el proceso evaluativo de las habilidades parentales, también hacen referencia a las Debilidades presentes, las cuales se dividen en tres niveles: Nivel Metodológico, Perspectiva Teórica y Recursos Profesionales.

A Nivel Metodológico surgen debilidades internas y externas de los/as evaluadores/as. Entre las internas se pueden identificar la escasez en la utilización de técnicas y/o instrumentos específicos de corte psicológico, debido a un déficit de éstos en el contexto chileno, aún cuando internacionalmente existe una cantidad considerable de instrumentos relacionados con esta temática. Es así como ante esta escasez, sólo es posible utilizar diversas herramientas, que si bien son 
validadas, no están diseñadas para esta temática en particular. Ahora bien, en cuanto a los instrumentos sociales, también existe un déficit en la utilización de técnicas 0 instrumentos que permitan sintetizar la información obtenida de manera objetiva, en distintos contextos evaluativos.

Como debilidades externas, se puede señalar la información incompleta, relacionada con las solicitudes emanadas desde el Tribunal, las cuales tienden a contener escasa información, quedando como tarea adicional para los/as evaluadores/as indagar en los antecedentes relevantes del caso o si existen otras causas judiciales asociadas, restando tiempo al análisis. Asociado a lo anterior, existe otra debilidad relacionada con los/as referentes a evaluar, los cuales son determinados, en primera instancia, exclusivamente por Tribunal, dificultando de esta manera el proceso, por cuanto al existir esta limitante judicial, se pueden dejar de lado a referentes que pueden ser significativos para el/la niño/a 0 bien considerar a referentes, que a priori, no pueden constituirse como tales para el cuidado de los/as niños/as generando una pérdida de tiempo, constituyéndose como otra debilidad, debido a que el número de referentes no puede ser sugerido por los/as profesionales. En la misma línea del tiempo otorgado para la evaluación, se presenta la inasistencia de los/as referentes a las sesiones, dificultando la recolección de información y en ocasiones, retrasando las audiencias, manteniendo al/a la niño/a en situación de vulnerabilidad. Finalmente, los recursos que están referidos a las condiciones de trabajo actuales, se relacionan con el lugar de evaluación (seeting) y el escaso recurso humano, dificultando la posibilidad de cumplir con las demandas solicitadas.

"Las debilidades del proceso... el tiempo para poder hacer las pericias... porque... es mucha la cantidad que se manda a hacer... y es muy poco el personal y el tiempo, por la cantidad de pericias que tenemos que hacer... es muy poco el tiempo para realizarlas. $Y$ otra situación que yo creo que igual podría es que las personas siempre llegan el ultimo día, a última hora... después que uno las ha citado muchas veces". (E6)
El segundo nivel, referido a la Perspectiva Teórica presenta una debilidad significativa, relacionada con la existencia de criterios indefinidos de habilidades parentales, generando discrepancias al momento de triangular la información tanto entre profesionales del área psicosocial como entre éstos últimos y el área jurídica, debido a la comprensión disímil del mismo fenómeno. Por lo tanto, en lo referente a la evaluación, la decisión sobre qué componentes evaluar y la definición de nivel de habilidad parental, queda a elección y criterio de los/as diferentes profesionales, pudiendo interferir en el grado de prolijidad del proceso.

El tercer nivel, relacionado con los Recursos Profesionales, se divide en dificultades técnicas y personales. Las técnicas referidas a las escasas instancias formativas, que se perciben como insuficientes tanto en cantidad como en calidad, lo que no permite una real actualización de contenidos referentes a las habilidades parentales. Las personales están ligadas al factor emocional, específicamente a la hiper-sensibilidad, característica que algunos/as profesionales señalan poseer, y que han aprendido a manejar, pero que igualmente es percibida como una debilidad, pudiendo sesgar los resultados.

\section{Sugerencias}

Desde la perspectiva de los/as profesionales en base a la experiencia que la práctica les brinda señalan en su totalidad que la evaluación de habilidades parentales está destinada a mejorar. Para ello entregan Sugerencias a nivel Metodológico, Teórico y Práctico.

A nivel Metodológico las sugerencias significativas van dirigidas a elaborar 0 estandarizar a la población chilena nuevas técnicas e instrumentos, tanto psicológicos y sociales, para realizar una evaluación específica y eficaz, destacando entre otros los inventarios, cuestionarios e instrumentos basados en la perspectiva y la opinión del/la niño/a, actualmente utilizada en el contexto internacional y que podrían constituirse como aportes relevantes para la especialización de la evaluación. Como otra sugerencia surge potenciar la tecnología creando una base de datos común donde los/as evaluadores/as de 
habilidades parentales, puedan tener acceso a las causas e informes permitiendo compartir $y$ agilizar la información entre las instituciones, además de promover la triangulación como herramienta de análisis.

A nivel Teórico, las sugerencias se orientan a la estandarización de parámetros, a través de la elaboración de criterios teóricos comunes para comprender el fenómeno de las habilidades parentales y permitir una evaluación más específica, enmarcada en un criterio de objetividad, permitiendo determinar adecuadamente la habilitación o inhabilitación $\mathrm{del} / \mathrm{la}$ referente evaluado/a. Con esto se pretende subsanar las discrepancias generadas en algunas ocasiones entre los/as profesionales del área psicosocial y los/as profesionales del área jurídica, disminuyendo la situación de vulnerabilidad de los/as niños/as. Por último, la generación de investigaciones que permitan elaborar teorías para mejorar la comprensión del concepto y aportar al progreso de la calidad de la evaluación.

\section{"[...] entonces la estandarización} de estos criterios, permitiría establecer los márgenes dentro de los cuales vamos a comprender una situación, una problemática y cómo vamos a evaluarla, y entonces la falta de estos márgenes genera este cuestionamiento, esta falta de validación y que en el fondo no es la validación del profesional lo que nos complica, sino como eso se traduce en la realidad que va a afectar a este niño, niña o adolescente [...]". (E2)

Por último a nivel Práctico, los/as profesionales sugieren un aumento de los recursos humanos, aumentando la dotación de duplas psicosociales dedicadas exclusivamente a la evaluación de habilidades parentales lo que supondría una optimización del tiempo para el proceso de evaluación y análisis de los diferentes casos. Como también, dentro de este nivel, que los requerimientos de evaluación de habilidades

que surgen como resultado de la experiencia práctica y la utilización de las competencias en el ejercicio parental, identificándolas como potenciables.

\section{"[...] las competencias parentales} son las que, potencialmente se puede parentales provenientes de Tribunal, consideren, en todos los casos, una evaluación del/la niño/a, debido a que se está evaluando las capacidades de un/a referente, en función de la relación que establece con el/la niño/a en un contexto determinado.

\section{Habilidades Parentales v/s Competencias Parentales}

En el contexto de la evaluación de habilidades parentales, aparece dentro de la revisión bibliográfica el concepto de Competencias Parentales, que abre otra arista en lo que respecta a la Definición. En este sentido, puede señalarse con respecto a las Habilidades Parentales, que los/as profesionales poseen una definición propia acerca del tema extrayendo medularmente, que están referidas a recursos y/o características que los/as distintos/as referentes del/la niño/a 0 adolescente debieran poseer para ejercer de manera adecuada los cuidados personales de éstos, constituyéndose como referentes protectores y afectivos significativos en su formación futura. Ahora bien, como componentes principales para la evaluación de habilidades parentales destacan en primer lugar el apego o vínculo, la capacidad empática, los estilos de crianza adoptados, la utilización de redes familiares y comunitarias; la salud mental, y los aspectos socioeconómicos. Así también, consideran la dinámica que se da entre los aspectos evaluados para el ejercicio adecuado de un rol parental.

Sin embargo, cuando al concepto de habilidades parentales se le suma el de Competencias Parentales, se producen dos líneas de análisis. La primera referida a la diferenciación, percibiendo a las competencias como recursos o capacidades intrínsecas del/la referente, tanto afectivas, cognitivas, entre otras; mientras que las habilidades son percibidas como los recursos y/o capacidades

tener, pero las habilidades es el resultado del desempeño que realmente se tiene en la práctica, 0 sea, una persona que tiene ganas de tener hijos, que es capaz de empalizar con las necesidades de otras personas, que puede posponer sus 
propias necesidades, [...], o sea, puede tener muchas competencias, que... pueden 0 no traducirse en habilidades parentales, las competencias son como los recursos personales emotivos, conductuales, afectivos que uno pueda tener, pero las habilidades son los resultados que tu logras en la práctica cuando eres padre [...]". (E1)

La segunda línea corresponde a las similitudes, considerada por parte de los/as profesionales, otorgándole sólo una diferenciación semántica más que teórica, ya que en la práctica son utilizados de manera indiferenciada en el ámbito psico-socio-jurídico.

“[...] En términos prácticos son 10 mismo, o sea, en términos prácticos no hay diferencias, es una cosa más de léxico que de cosa práctica digamos el termino. Es como, como poniéndoles un ejemplo, hace años atrás nosotros éramos un país subdesarrollado, hoy día somos un país en vías de desarrollo... seguimos siendo exactamente sub desarrollados, pero en términos más bonito [...]". (E3)

\section{DISCUSIÓN}

La presente búsqueda investigativa permite dar respuesta de manera óptima al objetivo central planteado, de manera que es posible describir el proceso de evaluación de habilidades parentales que utilizan los/as profesionales del ámbito del derecho de familia de la ciudad de Antofagasta, tal como se muestra en la Figura $\mathrm{N}^{\circ} 1$.

\section{FIGURA 1.}

Proceso de evaluación de habilidades parentales que utilizan los/as profesionales del ámbito del derecho de familia.

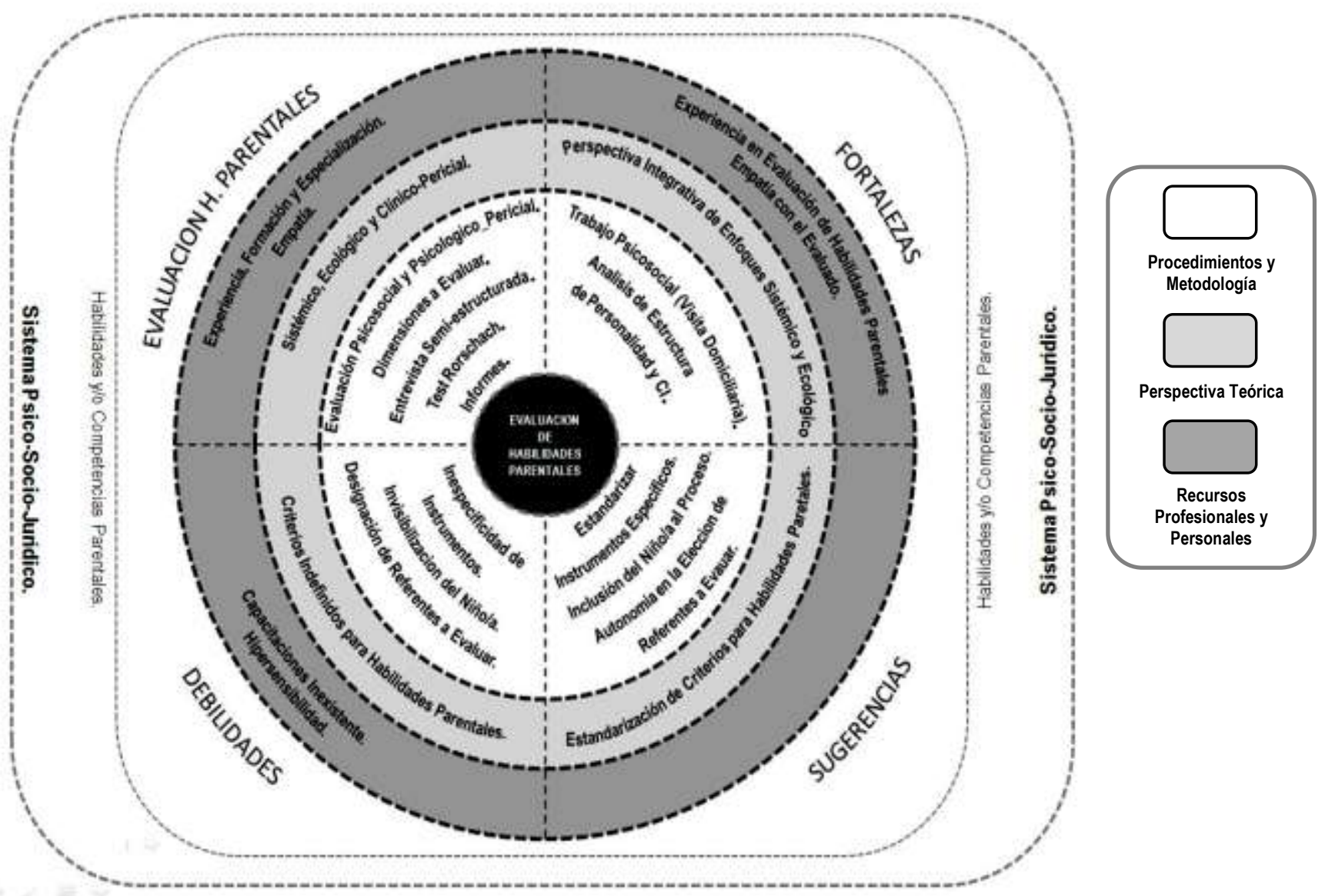


En este sentido, se puede señalar que aún cuando se trata de profesionales y/o instituciones que en la práctica pueden no tener una mayor consideración, se presenta la coyuntura de que en los discursos existen elementos comunes, que hacen suponer que si bien nunca se han dispuesto a establecer consensos respecto de esta práctica evaluativa, si se da la existencia de procedimientos, ideas, sugerencias símiles. Sin embargo, existen casos en los cuales se presentan diferencias significativas tanto en lo que respecta a elementos externos del proceso como a competencias del propio profesional.

En primera instancia es posible describir el aspecto metodológico del proceso, el cual puede ser abordado desde dos miradas, una de carácter psicosocial, percibida como una fortaleza en este tipo de procesos dado el carácter integral de este tipo de evaluación; como también otra de carácter psicológicopericial, la cual posee la fortaleza del análisis detallado de la estructura de personalidad y el C.I. del/la referente evaluado/a. Estas modalidades dependen tanto de los/as profesionales como de las instituciones en las que trabajan y que otorga los lineamientos para tal objeto. Por otra parte, dentro de este aspecto, se encuentra un elemento central en la práctica evaluativa y que dice relación con las dimensiones especificas para evaluar, entre las que destaca el apego como el componente más significativo de la evaluación, seguido de la empatía, los estilos de crianza, la plasticidad parental, el C.I., la salud mental, la utilización de redes comunitarias, y finalmente, el nivel socioeconómico, lo que coincide, en algunos aspectos, con los resultados de Garcia et al. (2007), difiriendo de éstos sólo en la evaluación de utilización de redes comunitarias y de la plasticidad parental. Sumado a lo anterior, estas dimensiones están estrechamente relacionadas con los planteamientos que Barudy (2005) utiliza en su guía de evaluación parental. Para lograr la evaluación de estas dimensiones, la entrevista semi-estructurada, emerge como la principal técnica de recolección de datos, coincidiendo con el contexto español, en el que esta técnica se constituye en el recurso básico en la evaluación de custodia (Rodríguez \& Ávila, 1999). Además dentro de la evaluación psicosocial, la visita domiciliaria destaca como una herramienta fundamental para realizar los análisis. Respecto a los instrumentos utilizados transversalmente está el Test de Rorschach el que permite identificar indicadores de personalidad que puedan interferir en el desempeño del/la referente, sin embargo, la bibliografía refiere que la utilización de Test Proyectivos, en otras zonas del país, es sólo de carácter complementario (Garcia et al, 2007). Es así que, a raíz de estas diferencias, surge como debilidad metodológica la escasa utilización de instrumentos específicos, tanto psicológicos como sociales, para evaluar habilidades parentales, relacionándose con la crítica que Bronchal (s.f.), realiza respecto a que la falta de estructuración del proceso invalida algunos instrumentos que son utilizados clínicamente, debido a su falta de especificidad. Teniendo presente lo anterior, es que surge de parte de los/as profesionales como principal sugerencia en este aspecto, la necesidad de crear y/o estandarizar a la población chilena distintos tipos de instrumentos específicos para habilidades parentales, que son utilizados en el contexto internacional.

Siguiendo en el ámbito metodológico, se encuentra que posterior a los análisis de los resultados obtenidos en la evaluación, se confecciona un informe con las conclusiones, el cual, en la ciudad, responde a tres tipos distintos: dos tipos de informes psicosociales, que difieren en algunos aspectos entre sí, sin embargo se asimilan en otros; y un informe psicológico, que no considera aspectos sociales del/la referente evaluado/a. Estas diferencias evidencian un déficit en la sistematización del proceso, dificultando el intercambio de información y posterior triangulación entre las instituciones tanto evaluadoras como judiciales, afectando negativamente la prolijidad del proceso y, en algunos casos, manteniendo la situación de vulnerabilidad del/la niño/a a la espera de un acuerdo. En este sentido, también se visualiza como debilidad la dificultad que tienen los/as profesionales para determinar qué referentes se evaluarán, ya que esta función es de exclusiva facultad del Tribunal, lo que conlleva a que se deje de lado al/a la niño/a en dichas evaluaciones, invisibilizándolo dentro del proceso; emergiendo de esta manera otra importante sugerencia por parte de los/as profesionales que guarda relación con el hecho de otorgar a los/as profesionales mayor libertad a la hora de escoger a los referentes a evaluar estipulando siempre, como procedimiento, una evaluación del/la niño/a lo que permitirá evaluar 
de mejor forma a los/as referentes y las dinámicas en relación a él/ella.

Por otra parte, importante es el hecho de considerar la base teórica en la que los/as profesionales se basan para realizar el proceso de evaluación de habilidades parentales, encontrando que éstos se adscriben a distintos enfoques teóricos. Entre estos enfoques se destacan el sistémico y ecológico, percibidos como una fortaleza dado el carácter integrativo, así como el clínico-pericial, los que están fuertemente relacionados con la visión de las instituciones en las que trabajan. Estos enfoques se condicen con los lineamientos que a nivel legislativo se otorgan a este tipo de instituciones, los que dan plena libertad a los equipos de trabajo de escoger las modalidades con las cuales deben cumplir sus objetivos (SENAME, 2007). Sumado a lo anterior, los/as profesionales identifican como debilidad significativa dentro del proceso, la existencia de criterios indefinidos para conceptualizar las habilidades parentales, dificultando su evaluación, debido a que la decisión queda a criterio de los/as profesionales, mermando la objetividad del proceso. En consecuencia, se hace necesario la estandarización de parámetros, que permitan a través de criterios comunes comprender las dimensiones significativas de las habilidades parentales, con el fin de determinar con mayor objetividad la habilitación o inhabilitación de un/a referente.

Respecto a los recursos personales con los cuales cuentan los/as profesionales, existe un consenso otorgando mayor valoración a la experiencia en comparación con la formación, considerándola como una fortaleza personal dentro del proceso de evaluación. Relacionado a lo anterior, se encuentra la presencia de la especialización que se adquiere con el trabajo práctico, el cual además se ve reforzado con capacitaciones, cursos y/o seminarios, los cuales a pesar de ser impartidos, siguen siendo insuficientes en la entrega de conocimientos más específicos, configurándose como una debilidad al no contar con información actualizada para realizar las evaluaciones.

Por otra parte la formación cobra importancia en cuanto a la adquisición de conocimiento teórico que los/as profesionales han obtenido en su formación de pre-grado, a esto se agrega los factores dentro de los cuales se considera la formación ética como una parte fundamental dentro del proceso, destacando la confidencialidad, donde si bien la información entregada no queda entre el/la referente y el/la evaluador/a dado el contexto judicial, se resguarda la información no relevante para el proceso. En cuanto a las características personales, no existe un consenso entre los/as profesionales sobre si juegan un papel importante al momento de evaluar, sin embargo, la empatía surge como fortaleza y factor relevante para establecer un buen rapport. En otro sentido, las características personales pueden convertirse en una debilidad para la evaluación, puesto que la hipersensibilidad presente en algunos/as profesionales interferiría en la objetividad del/la evaluador/a al momento de realizar la conclusión.

Es así como los hallazgos anteriormente mencionados se constituyen en un aporte significativo en la comprensión de esta temática a nivel regional, siendo posible identificar aspectos positivos y negativos, los cuales son percibidos como facilitadores y obstaculizadores del proceso de evaluación de habilidades parentales respectivamente. Así mismo, esta investigación se suma a los escasos estudios referentes a la temática dentro del sistema psico-socio-jurídico, tanto a nivel nacional como regional, aportando sugerencias que, en el contexto actual del derecho de familia, pueden establecerse como mejoras al proceso, colaborando con la optimización del trabajo evaluativo. Además, esta investigación, realizada desde una visión externa, se constituye en una instancia de retroalimentación para los/as profesionales que realizan la evaluación de habilidades parentales, cuya finalidad es aportar prolijidad al proceso actualmente realizado.

La desestructuración teórica visualizada anteriormente, también es percibida como una debilidad relevante dentro del proceso actual, según los profesionales entrevistados, dado que en Chile, al contrario de otros países, al no existir lineamientos estandarizados para la evaluación de habilidades parentales, con los cuales los/as profesionales orienten su proceso de forma común; insta a que este tipo de evaluación, las dimensiones específicas que conforman las habilidades parentales y la forma de evaluarlas, no queden estipuladas formalmente, quedando a criterio del/la 
profesional, como actualmente sucede. Cabe señalar que el establecimiento de estos lineamientos no dependería de forma directa de los/as profesionales, sino que de la creación de una legislación que regule este tipo de evaluación a nivel nacional a través de los organismos pertinentes como Ministerio de Justicia y SENAME, estableciendo criterios unificados, que den respuesta a las necesidades tanto del área psicosocial como del área jurídica que intervienen en este tipo de procesos.

En este sentido, los lineamientos deberían orientarse a determinar de manera clara, en primer lugar, qué se entiende a nivel legislativo por habilidades parentales, estableciendo criterios discriminatorios, para la toma de decisiones. Lo anterior se evidencia en un hallazgo que surge en la presente investigación, y que no fue considerado como objetivo en un principio, que se refiere específicamente a la conceptualización de las habilidades parentales versus las competencias parentales, en el que a nivel del sistema psico-socio-jurídico, se encuentran escasamente diferenciadas; evidenciándose profesionales que las identifican indistintamente, mientras que otros hacen claras diferencias entre estos conceptos, tal como ocurre en la literatura especializada, afectando en si el proceso evaluativo, dificultando la decisión de qué es lo más idóneo para evaluar, o qué es lo realmente modificable para un/a referente con un déficit en los recursos. Esta indefinición teórica, repercute en la metodología, debido a que los instrumentos utilizados son decididos por los/as propios/as profesionales, los que en la actualidad no son específicos para evaluar la temática.

El proceso de evaluación realizado en Antofagasta, en términos generales se presenta homogéneo, en cuanto a los procedimientos metodológicos y dimensiones teóricas, sin embargo, esto se ha logrado en base a la experiencia de los/as profesionales, más que por un marco regulatorio común. En este sentido, un aporte de esta investigación es hacer énfasis en aspectos de la evaluación que, en las condiciones actuales, son proclives a mejorar; tales como, incorporar en todos los ámbitos de evaluación de habilidades parentales el componente social, para poder obtener resultados tanto del/la referente como del contexto en el que se puede desarrollar el/la niño/a. También, la incorporación obligatoria de una evaluación del/la niño/a dentro del proceso, lo que contribuiría a identificar las dinámicas familiares dejando de invisibilizarlo/a, considerando su percepción dentro de la evaluación. Estandarizar un formato de informe único entre las instituciones evaluadoras también se constituye como un aspecto perfectible, colaborando con la triangulación expedita entre los distintos profesionales y operadores intervinientes en este contexto particular. Además se hace relevante la incorporación de nuevos instrumentos específicos estandarizados para la población chilena, ayudando a la objetividad y especialización del proceso. Y finalmente, lograr la unificación de criterios teóricos para definir habilidades parentales, generando un marco común entre las instituciones evaluadoras y las de justicia, que permita, por ejemplo, la creación de formatos de informes estandarizados, permitiendo el intercambio de información entre todos los/as operadores/as del sistema, con la finalidad de disminuir las discrepancias dentro del mismo.

En relación a las limitantes de la investigación, cabe señalar la imposibilidad de realizar el focus group inicialmente propuesto, que hubiese permitido contrastar la visión de los/as profesionales, enriqueciendo la recolección de información, en cuanto a la obtención de aspectos comunes. La unidad de análisis también se constituye como una limitante, debido a que estuvo compuesta mayoritariamente por mujeres, dada la proporción actual de profesionales, pudiendo sesgar los resultados, inclinándolos hacia una percepción femenina. También es relevante destacar la exclusión de peritos externos, debido a que éstos intervienen en escasas ocasiones. Otra limitante, que cabe señalar, es que los entrevistados pertenecen a sólo tres instituciones, pudiendo afectar su capacidad de autocritica.

Tras este estudio, se pueden abrir diversas líneas de investigación orientadas a la profundización de contenidos, a la percepción de otros/as operadores/as del sistema jurídico, ampliando la unidad de análisis, como también incorporando la visión de los mismos usuarios del sistema. También, resulta importante replicar este tipo de estudios en otras regiones del país, para conocer como se lleva a cabo 
este tipo de evaluación y comparar hallazgos entre sí; ya que al utilizar una metodología cualitativa se dificulta la generalización de los hallazgos obtenidos. $Y$ en base a lo anterior, comenzar con estudios orientados a la estandarización de nuevas técnicas e instrumentos, tanto psicológicos como sociales; además de la estandarización de los formatos de informes, con la finalidad de consensuar los aspectos más relevantes en cuanto a la metodología utilizada en este tipo de evaluación. Finalmente, la estandarización de criterios teóricos comunes de habilidades parentales se alza como el principal objetivo en futuros estudios, considerando para este propósito a todos los/as operadores/as del sistema psico-socio-jurídico, aportando a la comprensión homogénea del mismo fenómeno, perfeccionando el proceso, para así velar por el Bienestar Superior del Niño/a..

\section{REFERENCIAS}

Aguilar, G., (2008). El Principio del Interés Superior del Niño y la Corte Interamericana de Derechos Humanos. Centro de Estudios Constitucionales de Chile, Universidad de Talca, 6(1): 223-247. Extraído el 01 Abril, 2009 de http://www.cecoch.cl/htm/revista/docs/estu diosconst/revistaano_6_1.htm/Elprincipio1 1.pdf.

Asociación Chilena de Psicología Jurídica y Forense de Chile. (s.f.). Metodología a Aplicar dentro de la elaboración de un peritaje psicológico, en materia de cuidado personal. Autor. Extraído el 19 de Mayo, 2009

de http://www.colegiopsicologos.cl/peritaje.pdf

Barudy, J. (s.f.). Los Buenos Tratos en la Infancia y La Resiliencia Infantil en la Prevención de los Trastornos del Comportamiento. Conferencia del Dr. Jorge Barudy.

Barudy, J., Dantagnan, M. (2005). Guía de Valoración de las Competencias Parentales a través de la Observación Participante. Instituto de Formación Investigación e Intervención sobre la Violencia Familiar y sus Consecuencias.

Bronchal, J. (s.f.). Informes Periciales en Asuntos de Familia: Crítica Metodológica, Garantías para el Usuario y Propuestas para su Mejora.
García, F., Sir, G., Telchi, N. (2007). Escalas de Ackerman - Schoendorf para la Evaluación de la Custodia Parental (ASPECT) y Procesos Evaluativos en las Causas Relativas al Derecho de Cuidado Personal de Niños, Niñas y Adolescentes: Un estudio Exploratorio - Descriptivo. Proyecto de Tesis para optar al Título Profesional de Psicólogo y al Grado de Licenciado en Psicología. Escuela de Psicología, Facultad de Humanidades, Universidad de La Serena. La Serena, Chile.

Instituto Nacional de Estadísticas de Chile (2008). Población y Sociedad. Aspectos Demográficos. Santiago: Autor. Extraído el 01 de Abril, 2009 de http://www.ine.cl/canales/chile_estadistico/ demografia_y_vitales/demografia/pdf/pobla cion_sociedad_enero09.pdf

Krakow, E., (2007). Habilidades Parentales: La Importancia de las Conductas Parentales. Boletín Centro de Excelencia para el Desarrollo de la Primera Infancia, 6 (1): 23. Extraído el 31 Marzo, 2009 de http://www.excellenceearlychildhood.ca/documents/BulletinVol6N 01Mars07ESP.pdf.

Ministerio de Justicia de Chile (1998). Ley que Modifica el Código Civil y Otros Cuerpos Legales en Materia de Filiación, $\mathrm{N}^{\circ}$ 19.585. Biblioteca del Congreso Nacional. Extraído el 02 de Abril, 2009 de http://www.cejamericas.org/doc/legislacion/ cl-ley-filiacion.pdf

Ministerio de Justicia de Chile (2004). Ley que crea los Tribunales de Familia. No 19.968. Biblioteca del Congreso Nacional. Extraído el 02 de Abril, 2009 de http://www.bcn.cl/leyes/229557

Ministerio de Justicia de Chile (2005). Ley que Establece Sistema de Atención a la Niñez y Adolescencia a través de la Red de Colaboradores del SENAME, y su Régimen de Subvención, $N^{0} 20.032$. Biblioteca del Congreso Nacional. Extraído el 02 de Abril, 2009 de http://www.bcn.cl/leyes/240374

Otto, R., Edens, J. (2002). Parenting Capacity. En T. Grisso \& R. Borum (Eds.), Evaluating Competencies, Forensic Assessment and Instruments. Perspective in Law and Psychology, Vol. 16. $2^{\mathrm{a}}$ Ed.: Springer.

Peters, R. (2007). ¿Qué deben hacer los padres?. Boletín del Centro de Excelencia 
para el Desarrollo de la Primera Infancia, 6(1): 1. Extraído el 31 Marzo, 2009 de www.excellencearlychildhood.ca/document s/BulletinVol6No1Mars07ESP.pdf

Ramírez, T. Villarroel, M. (2006). Peritajes Psicológicos: Aproximación al proceso pericial de veracidad en casos de abuso sexual infantil, desde la perspectiva de peritos psicólogos de la ciudad de Antofagasta. Seminario de Tesis para optar al grado de Licenciado en Psicología, Escuela de Psicología, Universidad Católica del Norte, Antofagasta, Chile.

Reebye, P. (s.f.). Child Custody-Acces Evaluation: Cultural Perspectives. Extraído de

http://www.priory.com/psych/custody.htm.

Rodriguez, C., Ávila, A. (1999). Evaluación, Psicopatología y tratamiento en psicología Forense. Madrid: Fundación Universidad Empresa.

Ruiz, J. (2003). La Observación. Metodología de la Investigación Cualitativa. Bilbao: Universidad de Deusto.

Servicio Nacional de Menores de Chile SENAME (2007). Bases Técnicas de Línea Diagnóstica.

Servicio Nacional de Menores de Chile SENAME (2007). Competencias Parentales. Primera Infancia, Departamento de Protección de Derechos.

Servicio Nacional de Menores de Chile SENAME (2007). Lineamientos Técnicos Específicos, Modalidad Centros de Diagnóstico para Lactantes y Preescolares.

Zúñiga, M. (2003) Evaluación de niños en custodia. Boletín Sociedad de Psiquiatría y Neurología de la Infancia y Adolescencia. 14 (1), 48-52. 